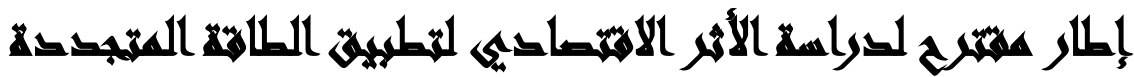

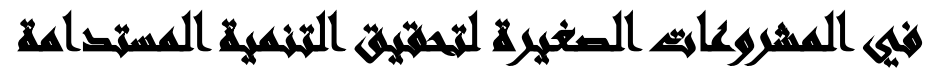

\section{[17]}

$$
\begin{aligned}
& \text { أحمد فؤاد مندور (')- نهال محمد فتحي الثحات(؟)- هانى عبد الفتاح عبد العظيم(r) } \\
& \text { أحمد محمد محمد الفالوجى الثحات الفي }
\end{aligned}
$$

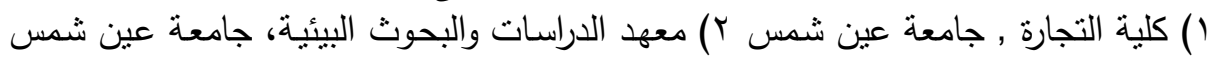

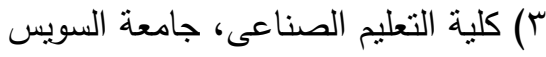

\section{المستخله}

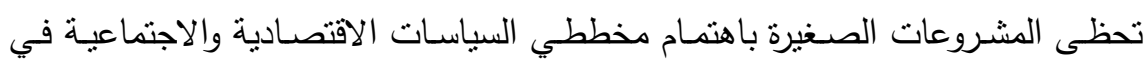

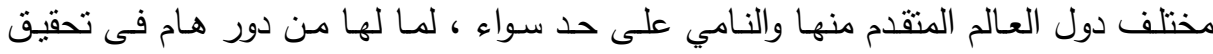
التمية الاقتصادية والاجتماعية.

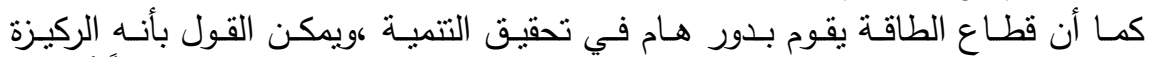

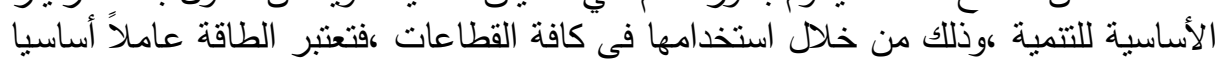

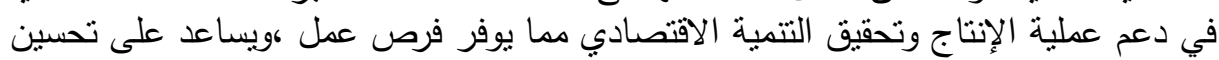
مسنتوى المعيشة بصفة الإنية عامة.

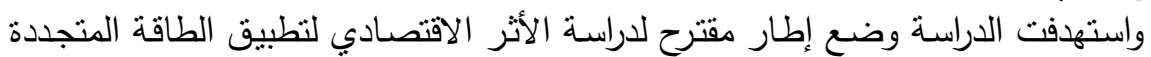

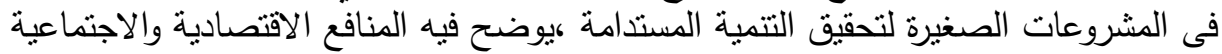

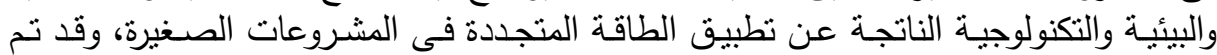

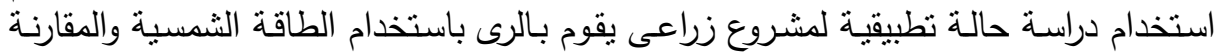
بينة وبين نظام بعمل بمولدات الديزل.

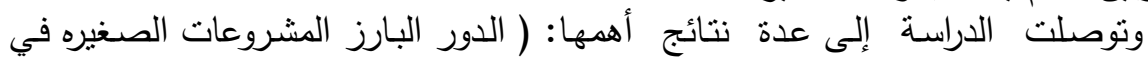

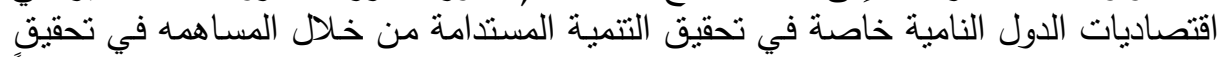

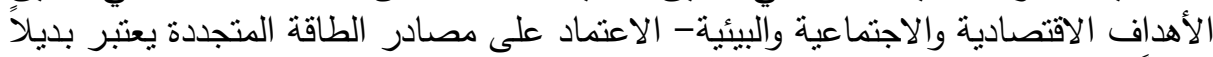

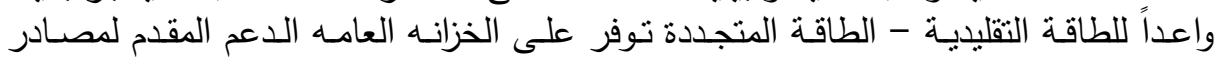

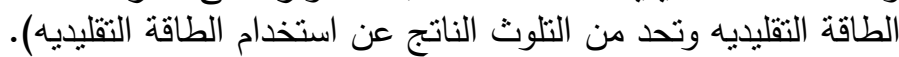

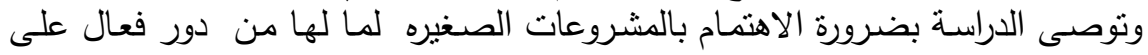

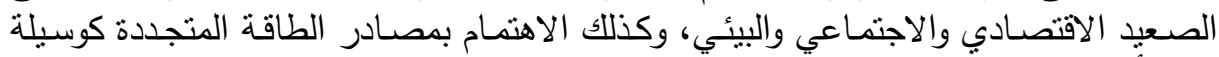

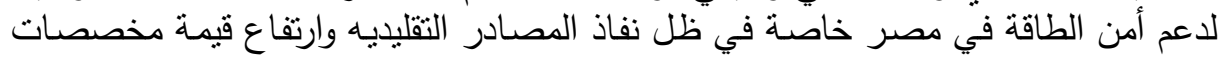

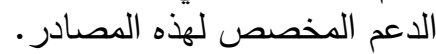
الكلمات المفتاحية: الطاقة المتجددة - المشروعات الصغيرة - التتمية المستدامة.

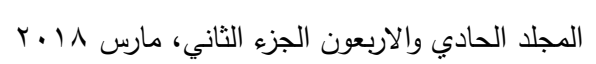




\section{المهابs}

تعتبر المشروعات الصغيرة ذات أهمية كبرى في كافة دول العالم وخاصة الدول النامية

منها، مع الأخذ بعين الاعتبار التفاوت النسبي الكبير بين المشروع الصغير في البلدان

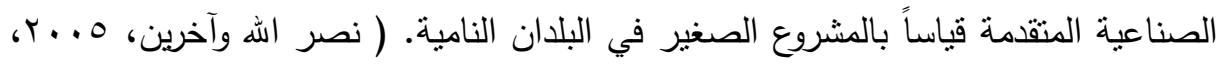
ص صمان كما أن الدول المتقدمة والنامية تضع صوب أعينها الاستفادة من المشروعات الصغيرة

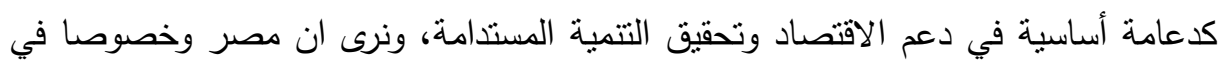
الآونة الأخيرة تسعى بكل السبل نحو الاستفادة من المشروعات الصغيرة اقتصادياً واجتماعياً

كما أن مصادر الطاقة المتجددة هي بشكل أساسي تلك المصادر التي لا تتضب في

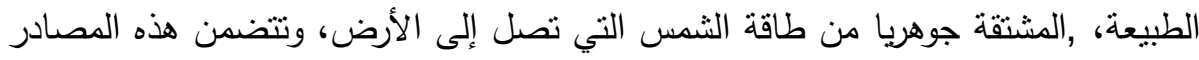

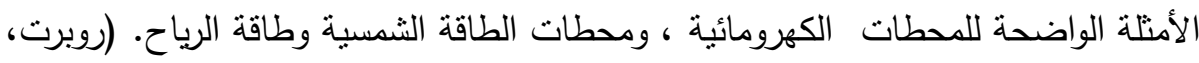

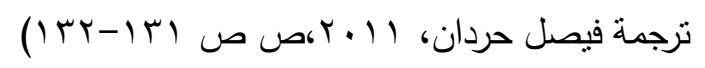
تساهم الطاقة المتجددة بشكل كبير في الحد من انبعاث الجسيمات والفلزات الضارة

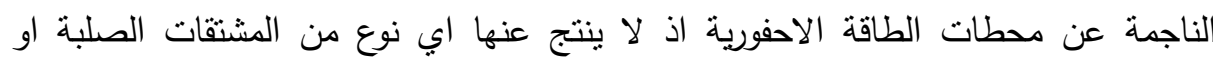

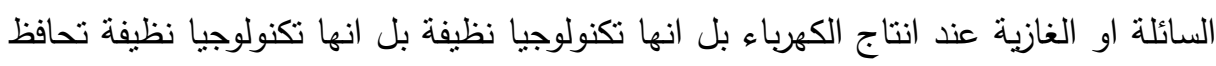

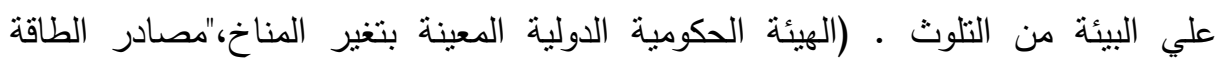

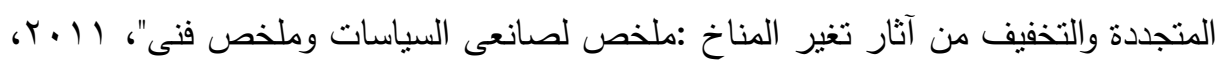
ص (10) وبالتالى فإن استخدام الشرروعات الصغيرة للطاقة المتجدة يساهم فى تحقيق التتمية الاقتصادية مع الحفاظ على البيئة مما يحقق الهدف النهائى وهو التتمية المستدامة. 


\section{التراسايت الماريخ}

ا - دراسة سامح عبد الكريم محمود أبو شنب( ( 1 • Y): بعنوان " دور المشروعات الصغيرة في معالجة مشكلتي البطالة والفقر"حالة الأردن" تهدف هذه الدراسة تقصني دور

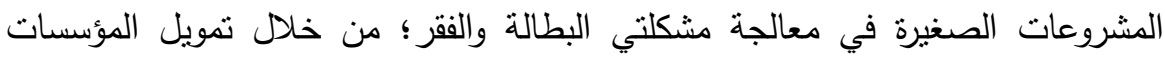

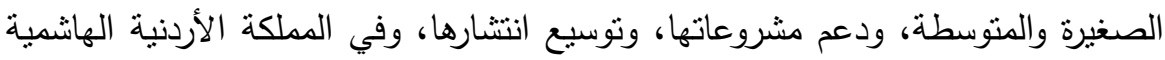
على وجه الخصوص، كما أنة يولى عناية خاصّة لدور المؤسسات المالية الإسلامية وصِيَغ التمويل الإسلامي في هذا المجال. وتوصل الباحث إلى أن للمشروعات الصغيرة دوراً فاعلاً في قدرتها على الحد من مشكلتي الفقر والبطالة.

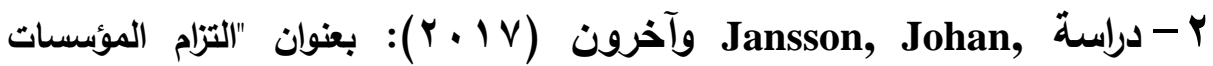
الصغيرة والمتوسطة بالاستدامة :تأثير التوجه الاستراتيجى والقيم الادارية تهدف هذه

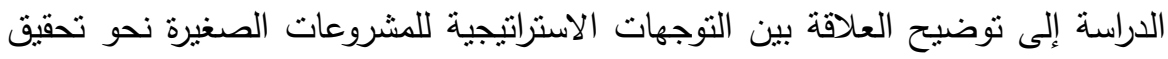

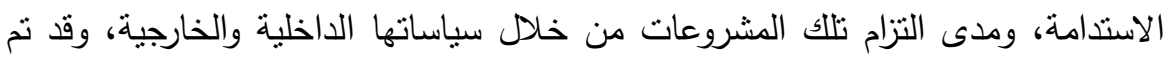
تحليل بيانات .0 0ـ شركة سويدية صغيرة ومتوسطة ونتج عن ذللك وضوح أهمية التزام

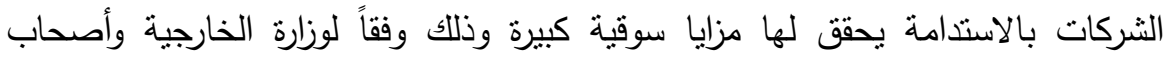

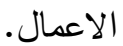

ومن أهم نتائج الدراسة أن اهتمام وزارة الخارجية وأصحاب الاعمال بمبادئ الاستدامة فى البيئة الداخلية والخارجية يحقق مزايا سوقية للمشروعات.

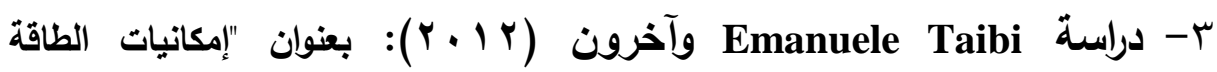
المتجددة فى التطبيقات الصناعية " تهدف هذه الدراسة إلى إمكانية استخدام الطاقة

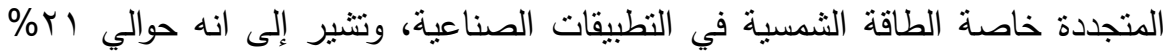
من الطاقة المستخدمة في الصناعات التحويلية ستكون من أصل متجدد بحلول عام •. . . وذللك بزيادة قدرها خمسة أضعاف المستويات الحالية، وبالتالي فيكون للطاقة المتجددة منافساً شرسا للطاقة المنتجة من الوقود الحفري. 
ويقترح الباحث وضع خارطة طريق مفصلة لاستخدام التكنولوجيا الحديثة من أجل

الاستفادة من مصادر الطاقة المتجددة خاصة الثمسية والحيوية منها.

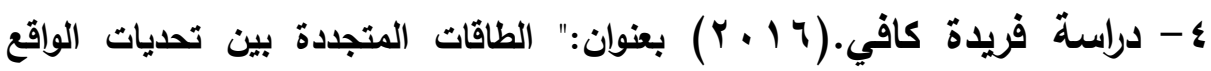

ومأمول المستقبل: التجرية الألمانية نموذجاً" تهدف هذه الدراسة إلى توضيح أهمية

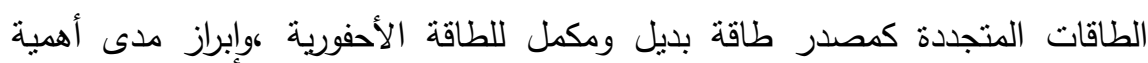

الاعتماد على الطاقات المتجددة لتحقيق تتمية حقيقة مستدامة ،ومعرفة تجربة ألمانيا في

مجال الطاقات المتجددة, واعتبارها تجربة رائدة في هذا المجال .

ومن أهم نتائج الدراسة :التوسع في استخدام مصادر الطاقة الأحفورية ودعمها، يعتبر

من أهم العوامل التي تعوق نمو الطاقة المتجددة وانتشارها.

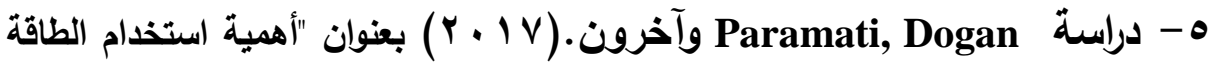

المتجدة فى الإنتاج الاقتصادى وحماية البيئة : دليل الدول النامية "تهذف هذه الدراسة

إلى بحث دور استهلاك الطاقة المتجددة على الاقتصاد وإنبعاثات ثانى اكسيد الكربون،

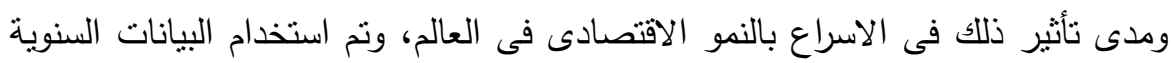

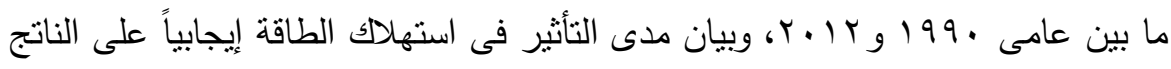

$$
\text { القومى، وسلبياً على إنبعاثات ثنانى اكسيد الكربون. }
$$

ومن أهم نتائج البحث هو الاقتراح على صانعى البعانيات الباسيات الاقتصادية، وتفعيل

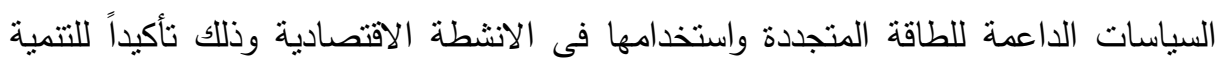

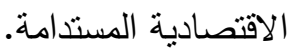

\section{and}

تتمنل مشكلة البحث فى أن استخدام المصادر التقليدية للطاقة ينتج عنة العديد من الملوثات والآثار السلبية على النواحى الاقتصادية وعلى البيئة أيضاً منل تغيرات المناخ

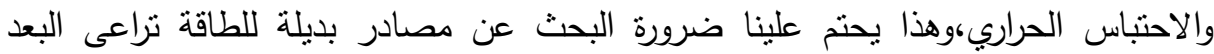


البيئي وتحد من تدهور البيئة،( الهيئة الحكومية الدولية المعنية بتغير المناخ "، II ب، ص 10 وبالتالي يمكن صياغة مشكلة البحث في صورة سؤال جوهري: هل يمكن عمل إطار

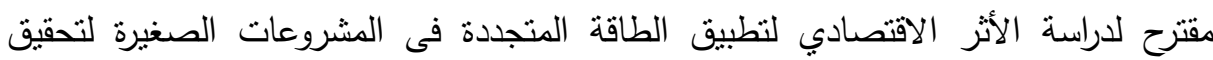

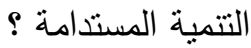

\section{ألهar}

يستمد البحث الحالى أهميته من وضع إطار مقترح لدراسة الأثر الاقتصادي لتطبيق الطاقة المتجددة بالمشروعات الصغيرة لتحقيق التتمية المستدامة وذلك من خلال: 1-بيان مدى إمكانية تطبيق الطاقة المتجددة في المشروعات الصغيرة. r-بيان أهمية استخدام الطاقة المتجددة لتحقيق التتمية المستدامة. r-الربط بين التتمية الاقتصادية وحماية البيئة.

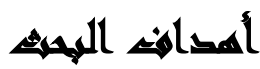

يهدف البحث إلى وضع إطار مقترح لدراسة الأثر الاقتصادي لتطبيق الطاقة المتجددة

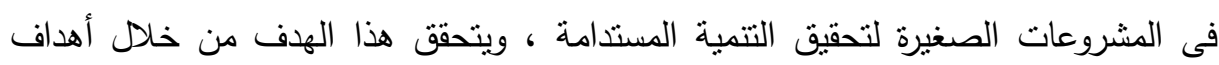

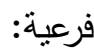

1-توضيح الأثر الاقتصادي لاستخدام الطاقة المتجددة على نمو المشروعات الصغيرة .

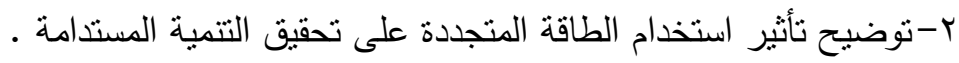
r-توضيح مفهوم الطاقة المتجددة وأنواعها المختلفة. ع- تحديد مفهوم التتمبة المستدامة وأبعادها. 0-وضع إطار مقترح لدراسة الأثر الاقتصادي لتطبيق الطاقة المتجددة بالمشروعات الصغيرة كوسيلة لتحقيق التتمية المستدامة. 


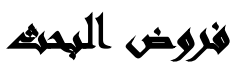

1- لا توجد علاقة معنوية ذات دلالة إحصائية بين استخدام الطاقة المتجددة في المشروعات

الصغيرة وتحقيق التتمية المستدامة.

ץ-لا نوجد علاقة معنوية ذات دلالة إحصائية بين استخدام الطاقة المتجددة ومراعاة البعد

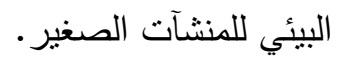

ץ- لا توجد علاقة معنوية ذات دلالة إحصائية بين استخدام الطاقة المتجددة وخفض تكاليف

الإنتاج وكذلك التكاليف البيئية.

ع- لا توجد علاقة معنوية ذات دلالة إحصائية تبين مراعاة المشروعات الصغيرة للبعد البيئي

وتحقيق التتمية المستدامة.

\section{منهمجي المهمه :}

قام الباحثّن بإتباع المنهج العلمي لتحقيق الهذف من بحثه كالتالي: منهج الدراسة وصفى تحليلي حيث قام الباحث بالدراسة النظرية، والدراسة العملية من أجل تحقيق أهداف البحث واختبار فروضة على النحو النالي:

أ- الدراسة النظرية: قام الباحثون بتجميع وتحليل وتفسير المعلومات المتعلقة بالعناصر

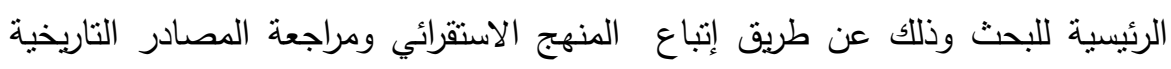

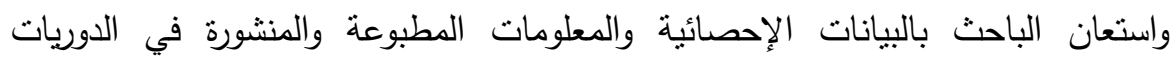
والمجلات المحلية ووضع إطار مقترح لتطبيق الطاقة المتجددة بالمشروعات الصغيرة لتحقيق التتمية المستدامة . ب- الدراسة العملية: قام الباحثون بدراسة حالة عملية لأحد المشروعات الزراعية التى

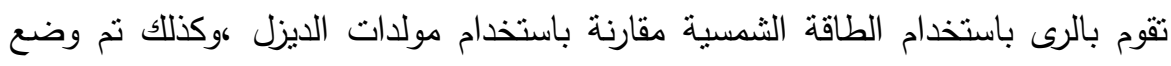
إطار مقترح يبين استفادة المشروعات الصغيرة من الطاقة المتجددة من اجل تحقيق التتمية بلهية

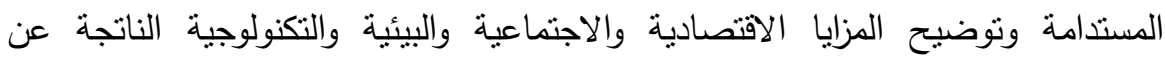

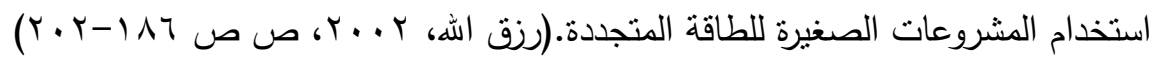




\section{هورض الصراسة}

حدود مكانية: تتمنل في المشروعات الصناعية الصغيرة العاملة فى جمهورية مصر العربية.

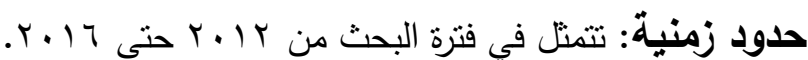
حدود بشرية: تتمنل في عينة من (مالكي المشروعات الصغيرة،العاملين بالمشروعات الصغيرة، العاملين بهيئة الطاقة الجديدة والمتجددة ).

\section{الاطار اللنظارى}

حتى يمكن تحقيق هدف البحث فقد تم تناوله في ثلاثة أجزاء على النحو التالي: الجزء الأول : تعريف المشروعات الصغيرة اهميتها الاقتصادية والاجتماعية. الجزع الثاني : مفهوم الطاقة المتجددة ومصادرها المختلفة وعلاقتها بالتتمية المستدامة. الجزء الثالث: دراسة الحالة والإطار المقترح لدراسة الاثر الاقتصادى لنطبيق الطاقة المتجدة فى المشروعات الصغيرة لتحقيق التتمية المستدامة. وسوف نتناول ذلك فيما يلي: - الجزء الأول: تعريف المشروعات الصغيرة اهميتها الاقتصادية والاجتماعية: لا يوجد تعريف محدد وموحد للمشروعات الصغيرة ، هذا بالإضافة إلى أن كلمة "صغيره "

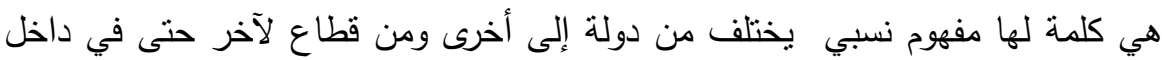

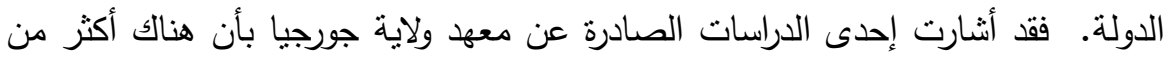

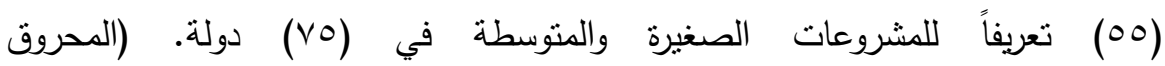

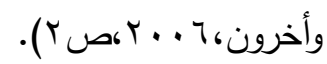

تعريف المشروعات الصغيرة في مصر: صدور القانون رقم اء| لسنة ع ...ب والمسمى بقانون تتمية المنشآت الصغيرة، وقام بوضع الإطار القانوني المنظم لتلالك المشروعات،وعرف القانون المشروعات الصغيرة بأنها " كل شركة أو منشأة فردية نمارس نشاطا اقتصاديا إنتاجيا أو خدميا أو تجارياً لا يقل رأسمالها المدفوع عن خمسين ألف جنيه ولا يجاوز مليون جنيه ولا

$$
\begin{aligned}
& \text { يزيد عدد العاملين فيها عن خمسين عاملا". (القانون المصرى رقم اء السنة ؟ . . ب). }
\end{aligned}
$$

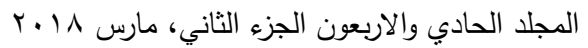


وتتمثل الأهمية الاقتصادية للمؤسسات الصغيرة في الأتي:

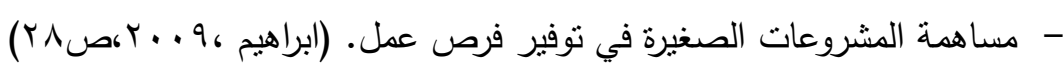

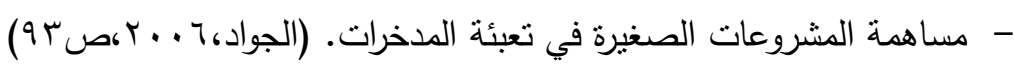

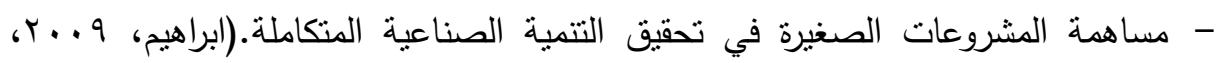

- مساهمة المشروعات الصغيرة في تحقيق التتمية الإقليمية.(ابوالسيد أحمد،ه. . ب، ص

\section{الأهمية الاجتماعية للمؤسسات الصغيرة في التنمية:}

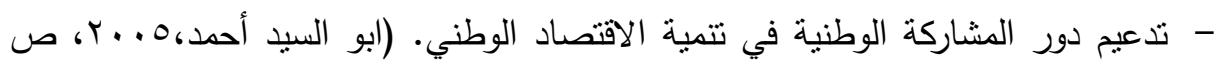

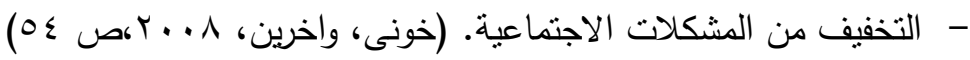

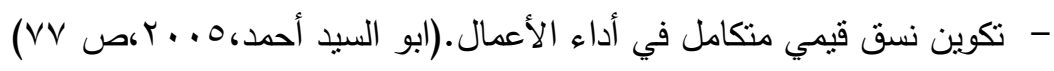

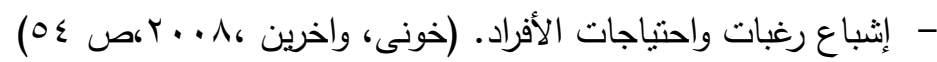

الجزء الثاني: مفهوم الطاقة المتجددة ومصادرها المختلفة وعلاقتها بالتنمية

المستدامة

1-مفهوم الطاقة المتجددة ومصادرها المختلفة: إن مصادر الطاقة المتجددة هي بشكل

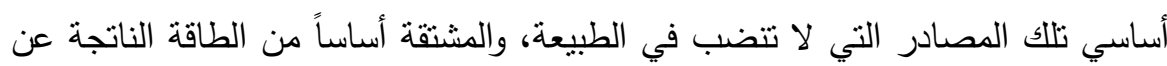
اشعة الثمس الني تصل إلى الأرض، وتتضمن هذه المصادر الأمثلة الواضحة الطاقة

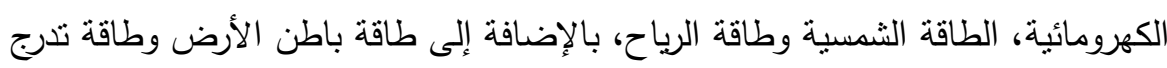
درجات حرارة المحيط، اللتين يتم اشتقاقهما من الكميات الكبيرة جدا من الطاقة الحرارية

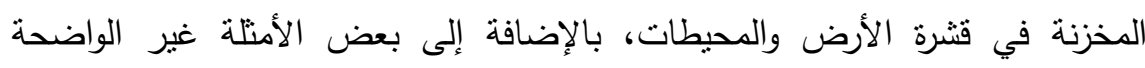

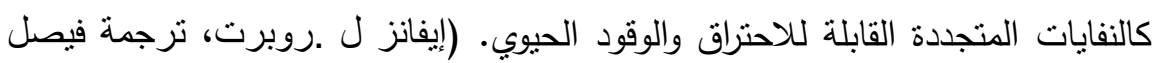

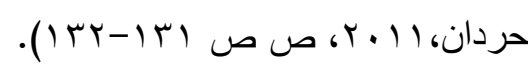


ץ-تعريف برنامج الأمم المتحدة لحماية البيئة(UNEP): الطاقة المتجددة عبارة عن طاقة لا يكون مصدرها مخزون ثابت ومحدود في الطبيعة، تتجدد بصفة دورية أسرع من وتيرة

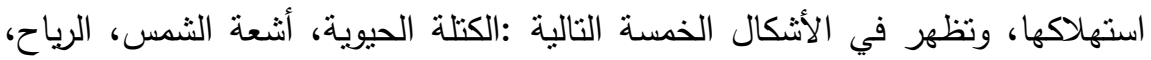
الطاقة الكهرومائية، وطاقة باطن الأرض. (موقع برنامج الأمم المتحدة لحماية البيئة

(.www.unep.org

وعليه فإن جميع مصادر الطاقات المتجددة منولدة عن مصادر الطاقات غير الأحفورية والتي لا تتضب أبدا وتتمنل في طاقة الشمس والرياح، طاقة الكتلة الحيوية، الطاقة الكهرومائية، طاقة باطن الأرض، وطاقة الأمواج والمد والجزر .وعليه فالطاقة المتجددة عبارة

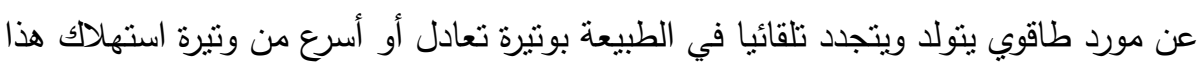

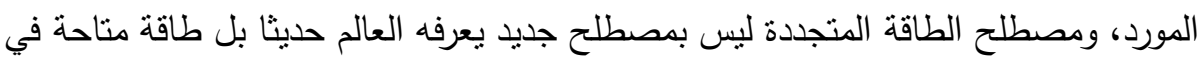
الطبيعة تم إحلالها على مدى قرون مضت بالطاقات الأحفورية Andexer Thomas ) . ,2008,P16) r-أهمية الطاقة المتجدة: نشكل الطاقة المتجدة المصدر الرئيسي للطاقة العالمية خارج الطاقة التقليدية وهناك اهتمام عالمي كبير بها كمصدر مسنقبلي للطاقة ، بحيث تكون بديلا للطاقة الأحفورية والتي تسعى عديد من الدول وخاصة الصناعية منها إلى استبدالها

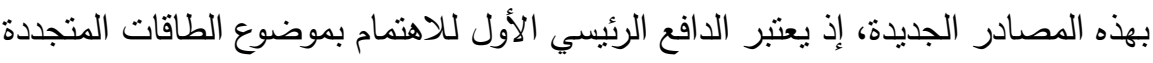

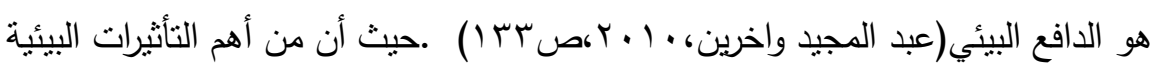
المرتبطة باستخدامات الطاقة ما يعرف بظاهرة الاحتباس الحراري ، وعلى العكس من ذلك

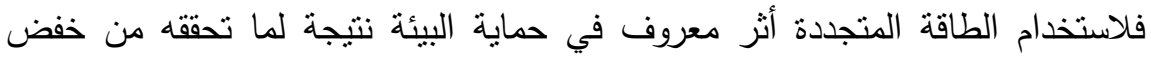

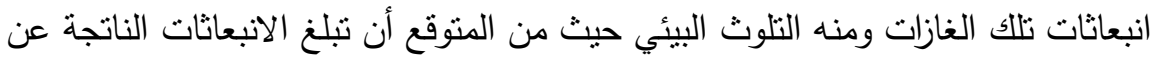

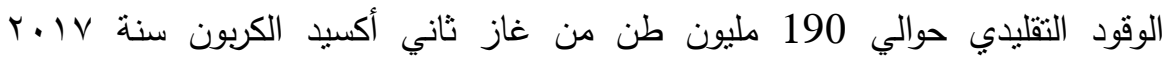

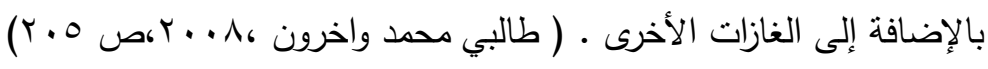
ـ-مفهوم التنمية المستدامة: وقد تم تعريف التنمية المستدامة أثناء انعقاد مؤتمر الأرض

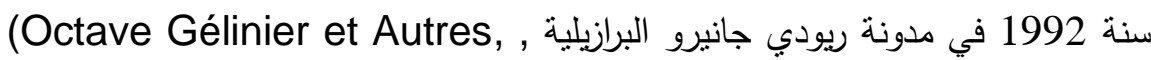
France, 2005, P.22).

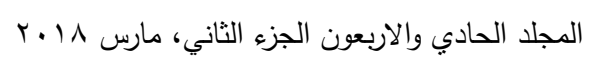


ويمكن تعريف التتمية المستدامة بأنها " تتمية توفق بين التتمية البيئية والاقتصادية والاجتماعية فتتشأ دائرة صالحة بين هذه الأقطاب الثلاثة، فعالة من الناحية الاقتصادية، عادلة

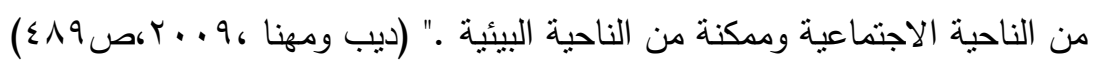
ه - علاقة التنمية المستدامة بالطاقة: أ- الطاقة وجذول اعمال القرن ابِ أقر مؤتمر الامم المتحدة (unced) المعني بالبيئة

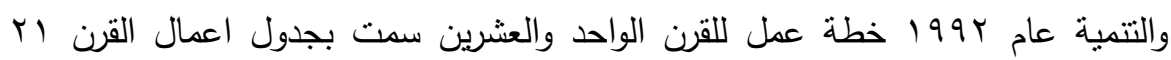
حيث تتاول قضايا الطاقة في اجزاء عديدة من الخطة كانعكاس لأهميتها وقيمتها كمدخل ضروري في عمليات واهداف التتمية المستدامة. الأول يتضمن المسائل المتعلقة بالتنمية الاقتصادية والاجتماعية خاصة ما يأتي: (تخفيف وطأة الفقر - تغيير انماط الانتاج والاستهلاك - نتميه المسنوطنات البشرية)

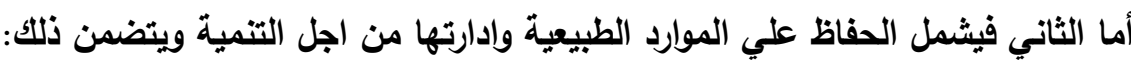

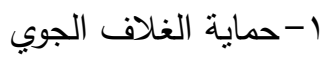
r- دفع وتعزيز التتمية الزراعية وتحسين الانتاجية في الناطق الريفية وتسعي الاهداف

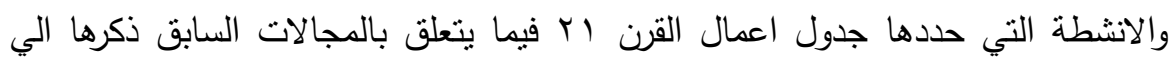

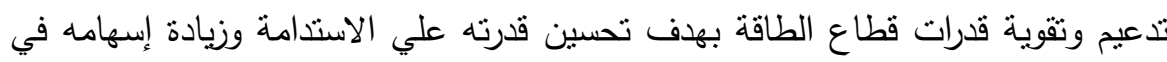
تحقيق التنمية المستدامة في القطاعات الاخرى. ب- لجنة التنمبة المستدامة التابعة للأمم المتحدة في دولتها الحية الحادبة عشر المنعقدة في

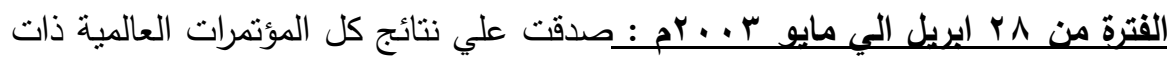

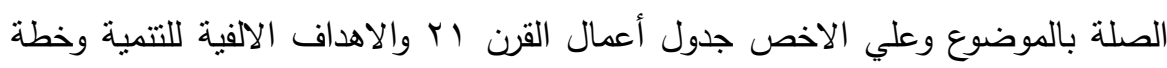
التتفيذ نتائج مؤتمر القمة العالمي للتتمية المستدامة علي العلاقة الوثيقة بين الطاقة الطية والاهداف الجوهرية للتتمية المستدامة وأهمها التخفيف من وطأة الفقر وتغير الانماط غير

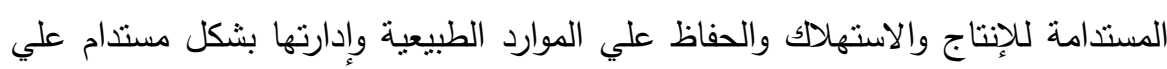

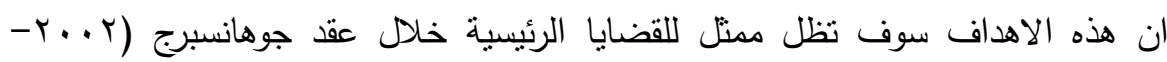




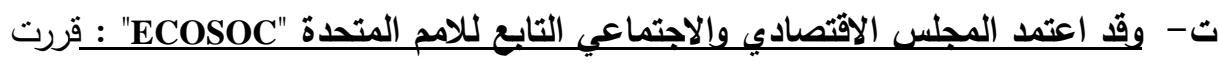
الجولة الحادية عثر للجنة التتمية المستدامة بأن تكون الطاقة هي الموضوع الرئيسي لدورة

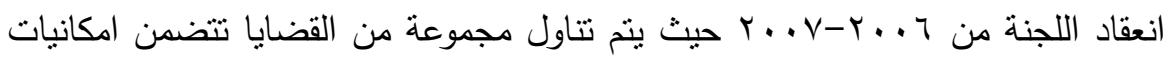
الوصول للطاقة وكفاءة الطاقة والتغير المناخي وتتوع مصادر الطاقة بما في ذللك

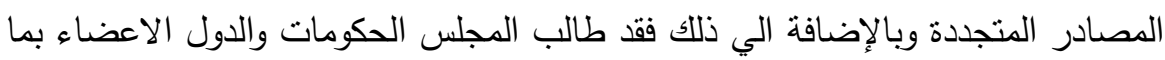

(1) وضع استراتيجيات وطنية للتنمية المستدامة واتباع نهج اكثر شمولا في تتفيذها .

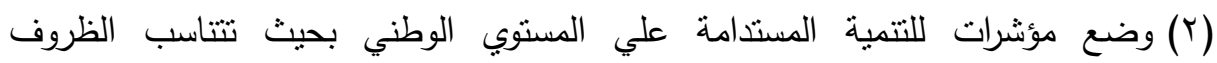

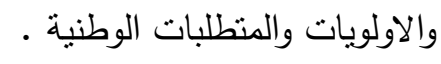

(r) إعداد نقارير وطنية تركز علي التقدم الملموس في التطبيق ونتثمل المنجزات والمعوقات

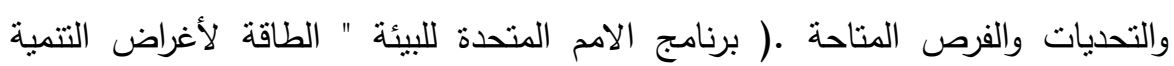

$$
\text { الجزء الثالث: دراسةة الحالة والاطار المقترح: المنقة العربية "، ص ص ه-7). }
$$

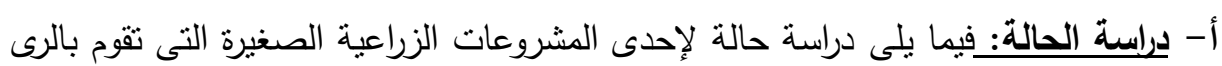
باستخدام الطاقة الثمسية وبيان الاثز الاقتصادى لاستخدام الرى بالطاقة الثمسية مقارنة

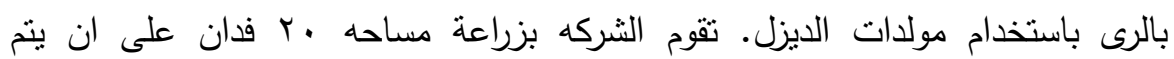

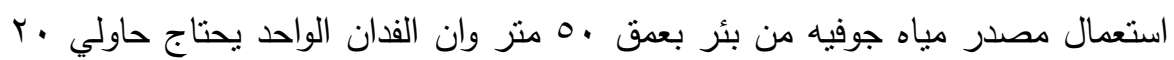

\begin{tabular}{|c|c|}
\hline القيمة & بيانات المولا المستخدم \\
\hline • ب كيلو وات & قدرة المولد \\
\hline |....... & السعر بالجنية المصرى \\
\hline ع ساعات & عدد ساعات العمل لكل • ب لتر سولار \\
\hline ا & السعر الرسمى للتر السولار \\
\hline 0 & متوسط سعر لتر السولار شامل النقل \\
\hline . . 0 جنية & تكلفة تغيير الزبت للمولد لكل • rا ساعة نشغيل \\
\hline 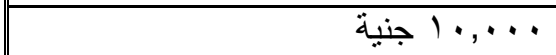 & تكلفة عمل عمرة لموتور المولا كل ؛ سنوات \\
\hline
\end{tabular}

$$
\text { متر مكعب من المياه يوميا. }
$$

أولا: تكاليف التشغيل بيولدات الدبزلِ التكاليف الثابتة والمتغير للتشغيل بمولدات السولار

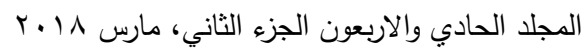


وان عدد ساعات الري V ساعات يوميا باستخدام مولا قدرته . r حصان فان الاحتباج

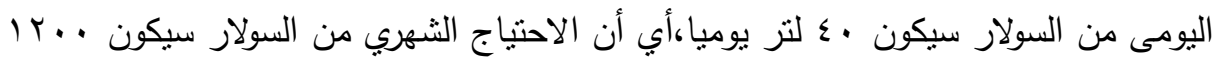

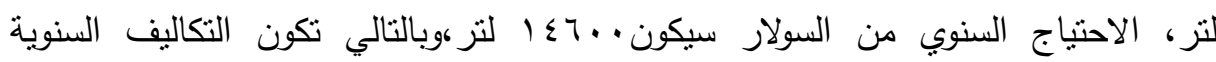
للتشغيل بالسولار = . . . ثانياً: التشغيل باستغد/م الطاقة الشعسية: منوسط تكاليف تشغيل حصان واحد بالطاقة

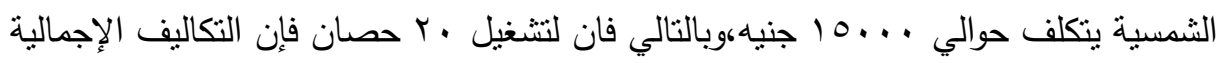

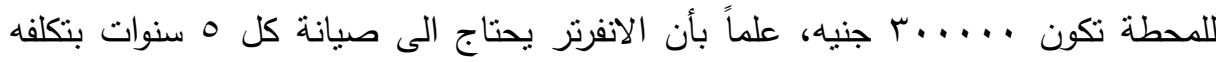

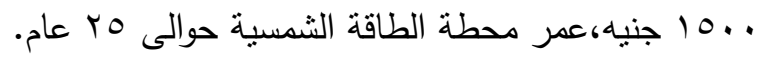

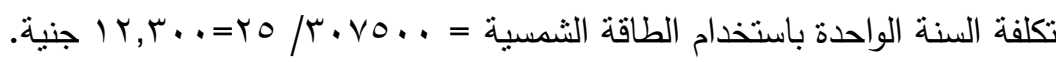

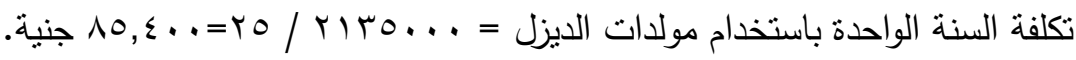
اى ان تكاليف التشغيل بالديزل سنويا تعادل ما يقرب من سبعة اضعاف تكاليف التشغيل

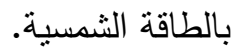
جدول: يوضح المقارنة بين تكاليف تشغيل مولدات الديزل ومحطة طاقة شمية

\begin{tabular}{|c|c|c|}
\hline الطاقة الشمسية & مولدات الديزل & 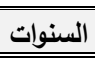 \\
\hline ونشغيل وحدة الطاقة الثمسية تكاليف إنشاء & 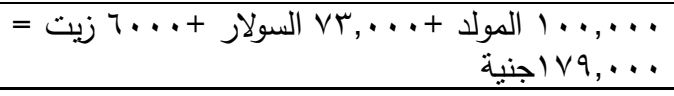 & 1 \\
\hline ....... & جنية $\vee \vee, \ldots=\uparrow, \ldots+\vee r, \ldots$ & r \\
\hline ........ & جنية & $r$ \\
\hline ........ & 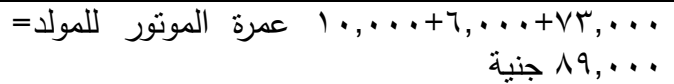 & $\varepsilon$ \\
\hline 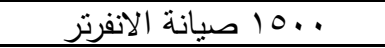 & جنية $\vee q, \ldots=7, \ldots+\vee r, \ldots$ & 0 \\
\hline ....... & جنية & 7 \\
\hline ........ & ج $\vee q, \ldots=7, \ldots+v r, \ldots$ & $\mathrm{V}$ \\
\hline ….... & 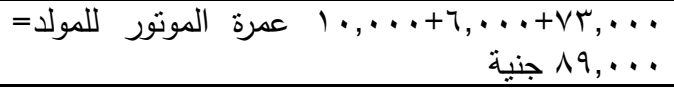 & $\Lambda$ \\
\hline ........ & 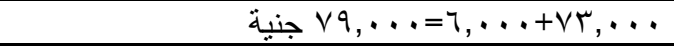 & 9 \\
\hline 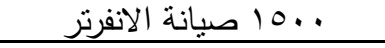 & جنية $\vee \vee, \ldots=7, \ldots+\vee r, \ldots$ & $1 \cdot$ \\
\hline ....... & جنية $\vee \vee, \ldots=7, \ldots+\vee r, \ldots$ & 11 \\
\hline c...... & 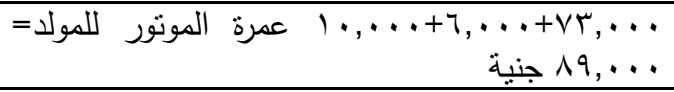 & IT \\
\hline & جنية & $1 \pi$ \\
\hline
\end{tabular}


أحمد فؤاد مندور وآخرون

\begin{tabular}{|c|c|c|}
\hline الطاقة الشمسية & مولدات الايزل & 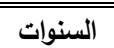 \\
\hline ....... & . & $1 \varepsilon$ \\
\hline ل . 10 صيانة الانفرثر & . & 10 \\
\hline$\ldots \ldots$ & 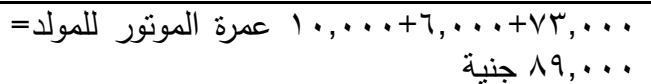 & 17 \\
\hline ........ & جنية $\vee \vee q, \ldots=\uparrow, \ldots+\vee r, \ldots$ & IV \\
\hline ....... & 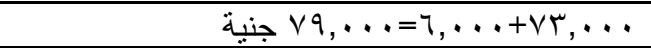 & 11 \\
\hline ........ & (ن $\vee q, \ldots=7, \ldots+\vee r, \ldots$ & 19 \\
\hline ل . 10 صيانة الانفرتز & 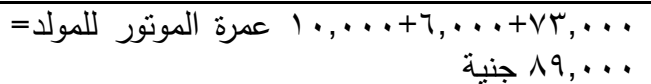 & r. \\
\hline$\ldots \ldots$. & جنية $\vee \vee q, \ldots=7, \ldots+\vee r, \ldots$ & YI \\
\hline$\cdots \cdots \cdot$ & . & rt \\
\hline$\cdots \cdots \cdot$ & جنية & r \\
\hline ....... & 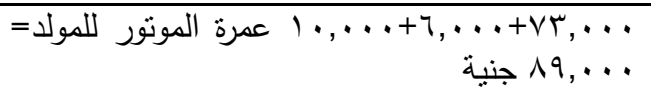 & $r \leq$ \\
\hline . . 10 صبانة الانفرتز & ( $\vee 9, \ldots=7, \ldots+V r, \ldots$ & ro \\
\hline$r \cdot v, 0 \ldots$ & $r, 1 r_{0}, \ldots$ & 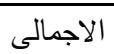 \\
\hline
\end{tabular}

- التكاليف الاستثمارية الأولية لمولدات الديزل اقل من التكاليف الاستثمارية الأولية للنظام

الثمسي.

- - تكاليف مولدات الديزل يعادل ستة اضعاف النظام الثمسي بعد مرور 0 سنه التي تمنل العمر الافتراضي في النظام الشمسي.

- استخدام خلايا الطاقة الثمسية نظام ذو جدوى اقتصاديه على المدى الطويل. ب- وضع الاطار المقترح: تعتبر الطاقة المتجددة أحد أهم مصادر الطاقة الحاليه في ضوضه مفهوم التتمية المستدامة لما لها من أهميه في توفير مصادر طاقه بديله متجددة خاصة الهال

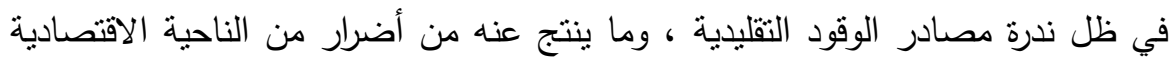
والاجتماعية والبيئية.

وتتمثل أهمبه الطاقة المتجددة في ما بليِي: (الطاقة المتجددة لا تتفذ- الطاقة المتجددة تعطي طاقة نظيفة خالية من الملوثات-الطاقة المتجددة نحصل عليها من البيئة الطبيعية المحبطة -

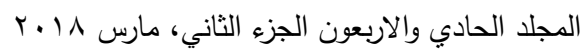


الطاقة المتجدة تأمين فرص عمل جديدة - الطاقة المتجددة تحد من الإنبعاثات الغازية

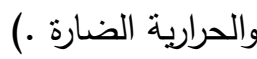

كما ان المشروعات الصغيره تحتل مكانه بارزه في اقتصاديات الدول النامية وذللك نتيجة

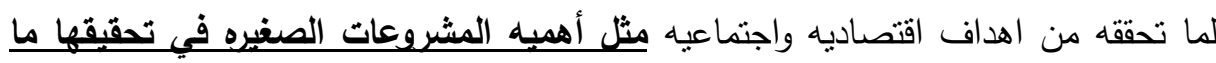
يلي: (توفير فرص عمل - تتمية الصادرات -تحقيق التتمية المتكاملة - تحقيق التتمية

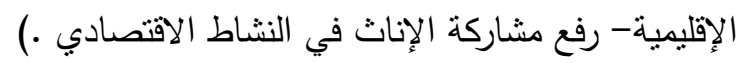

وفيما يلي الاطار المقترح الذي يراه الباحثين لدراسة الأثر الاتتصادي لتطبيق الأني الطاقة المتجددة في المشروعات الصغيره لتحقيق التنمية المستدامة والذي يتكون من: أولاً: التتمبة الاقتصادية: ولتحقيق التتمية الاقتصادية يرى الباحثون ما يلي:

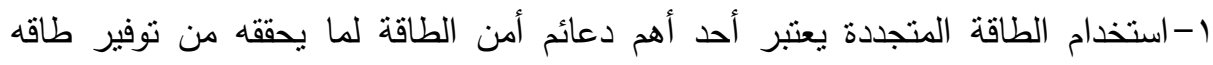

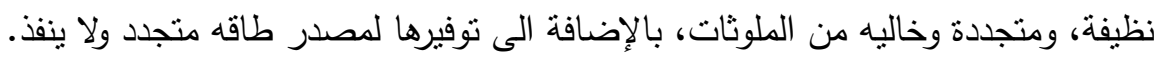

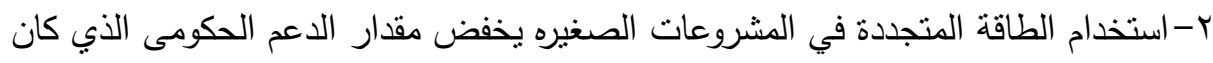

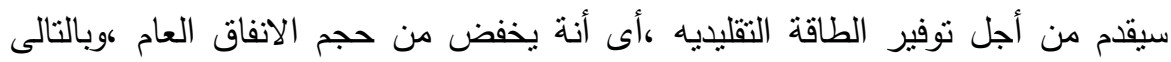
يخفض عجز الموازنة العامة للدولة . r- استخدام الطاقة المتجددة في المشروعات الصغيره يخفض استيراد المواد البترولية ويعتمد على موارد الطاقة المتجددة والمحلية وبالتالى يخفض من عجز الموازنة العامه. ع- استخدام المشروعات الصغيره من مصادر الطاقة المتجددة يخفض من التكاليف البيئيه

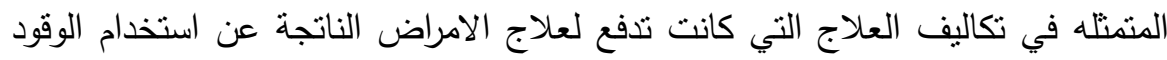
التقليدى وما ينتج عنة من ملوثات ضارة بالصحة والبيئة.

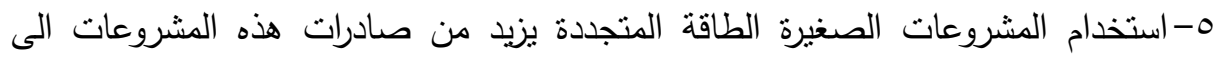
الدول التى نتشرط مراعاة البعد البيئى فى وارداتها. 
ثانبياً : التنمبة الاجتماعية:ولتحقيق التتمية الاجتماعية يرى الباحثون ما يلي:

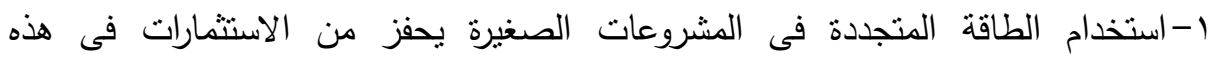

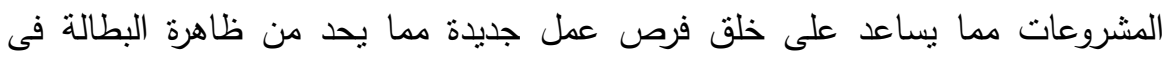
المجتمع وبالتالى يحد من الآتار السلبية لها ،مثل خفض معدلات علئ الجريمة. ץ-استخدام المشروعات الصغيرة للطاقة المتجددة يُعبر عن مدى اعنراف هذه المناف المشروعات بالمسؤولية الاجتماعية مما يُحسن من صورة هذه المشروعات في المجتمع. r-استخدام المشروعات الصغيرة للطاقة المتجددة يُحسن من الصورة هورة الذهنية لهذه المشروعات ويساعدها فى تحقيق أهدافها المتعلقة بالمجتمع المحيط بها. ع-استخدام المشروعات الصغيرة للطاقة المتجددة يساعد هذه المشروعات على استخدام الموارد الموجودة بالبيئة المحلية والمنمثلة فى مصادر الطاقة المتجددة وعدم الاعتماد على لهـ الواردات.

ه-استخدام المشروعات الصغيرة للطاقة المتجددة يحد من الفقر حيث يساعد على نتمية

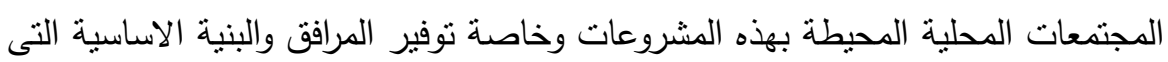
تساعد على رفع مستوى المعيثة لأبناء هذه المنطقة.

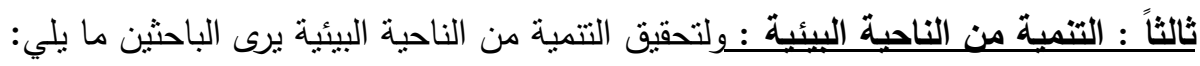
1-استخدام المشروعات الصغيرة للطاقة المتجددة بحد من الملوثات الناتجة عن استخدام مصادر الطاقة التقليدية. r-استخدام المشروعات الصغيرة للطاقة المتجددة يشجع الإتتاج الأنظف، ويعمل على نشر

$$
\text { المشروعات الخضراء صديقة البيئة . }
$$

r- استخدام المشروعات الصغيرة للطاقة المتجددة يساهم فى الحد من ظاهرة التغيرات المناخية وبالتالى يساعد على تحسين المناخ والحد من ظاهرة الاحتباس الحرارى. ع - استخدام المشروعات الصغيرة للطاقة المتجددة يُحسن من وضع مصنس مصر ضمن نرتيب الدول

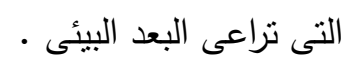


رليعا: التنمبة من الناحية التكنولوجية: ولتحقيق التتمية من الناحية التكنولوجية يرى الباحثون ما يلي: 1-تغيير التوجه التكنولوجي نحو استخدام الطاقة المتجددة كبديل أكثر أمناً وأكثر نطويراً في المستقبل لدي المستثمرين وخاصة الصغار منهم. بونه r-وضع خطط وبرامج تهدف إلى الوصول لمجتمع المعرفة حيث يتم دمج تكنولوجيا لخدمة إستراتيجيات التتمية المستدامة.

r-بناء المدن الذكية التي تعتمد على التكنولوجيا المتطورة وتستخدم طاقة متجددة منعدمة الإنبعاثات.

ع-نقل التكنولوجيا وأسرارها من الدول المتقدمة وذلك من خلال الاحتكاك مع الخبراء والاستشاريين الدوليين في مجال الطاقة المتجددة والاستفادة من الخبرة الدولية فى هذا المجال.

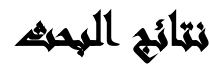

من أهم النتائج التي توصل إليها البحث ما يلي:

1- هناك دوراً بارزاً للمشروعات الصغيره في اقتصاديات الدول النامية باعتبارها قاطرة التتمية والملاذ الأخير في اقتصاديات نلك الدول.

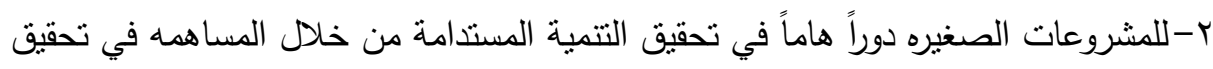
الأهداف الاقتصادية والاجتماعية والبيئية. r-استخدام المصادر التقليديه للطاقة يزيد من حجم الانفاق العام متمثلا في زيادة الإئه الدعم

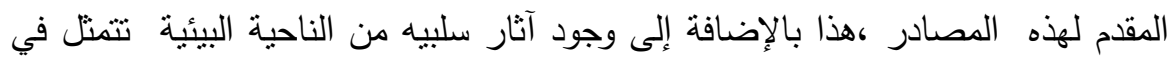
زيادة حجم الملوثات الناتجة عن استخدام المصادر التقليديه للطاقة.

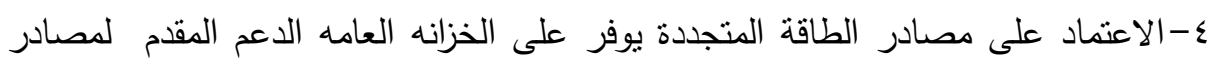
الطاقة التقليديه بالإضافة إلى الحد من التلوث الناتج عن قطاع المحروقات كل هذا بالإضافة إلى خفض التكاليف اللازمة لتوفير العلاج للأمراض الناتجة عن استخدام الطاقة التقليديه والملوثه للبيئة حيث أن الطاقة المتجددة تعتبر بديلاً نظيفاً وصديق للبيئة.

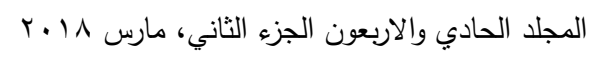


ه-استخدام الطاقة المتجددة في مصر يحتاج إلى سياسات واعية وهذا ينطلب السعى نحو

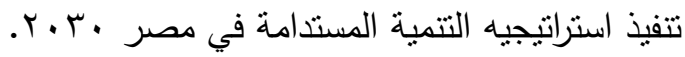

\section{التموسياهت}

$$
\text { في ضوء ما تم عرضه سابقاً يوصي البحث بالاتي: }
$$

1-يجدر بمصر الاهتمام بالمشروعات الصغيره لما لها من دور فعال على بلى الصعيد

$$
\text { الاقتصادي والاجتماعي والبيئي. }
$$

ץ-ينبغى زيادة الاهتمام بمصادر الطاقة المتجددة باعتبارها وسيلة لاعم أمن الطاقة في مصر

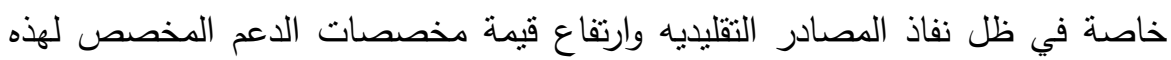

$$
\text { المصادر من الموازنة العامة. }
$$

r-ضرورة دعم المشروعات الصغيره الصديقه للبيئة وخاصة تلك التي تعمل بالطاقة

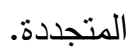

ع-الاهتمام بسن القوانين المنظمه لتشغيل للمشروعات الصغيره بالطاقة المتجددة مع تقديم

$$
\text { حوافز بيئية للمشروعات التى تحقق نوافق بيئى. }
$$

ه-العمل على تشجيع الاستثمارات في مجال الطاقة المتجددة مع تقديم الدعم للمشروعات

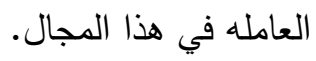

\section{zall}

الهيئة الحكومية الدولية المعينة بتغير المناخ،"مصادر الطاقة المتجددة والتخفيف من آثار تغير

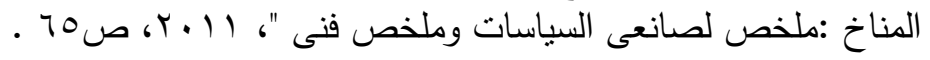

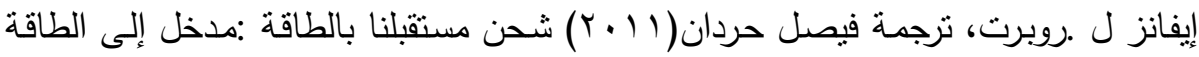

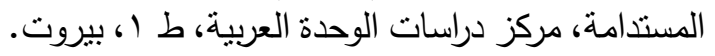

برنامج الامم المتحدة للبيئة ( الطاقة لأغراض التتمية المستدامة في المنطقة العربية )إطار

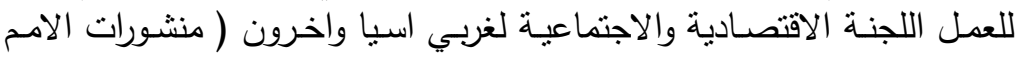

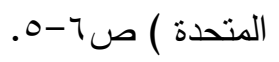

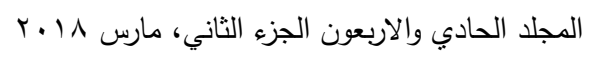




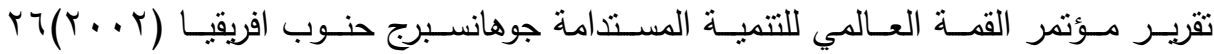

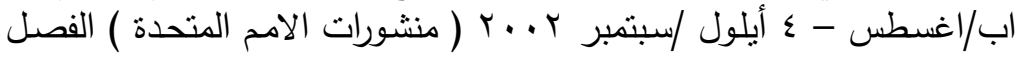
الاول

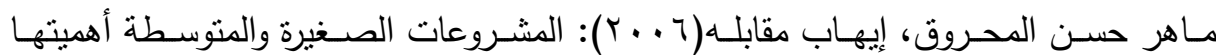
ومعوقاتها، مركز المنشآت الصغيرة والمتوسطة ، الأردن الصنات

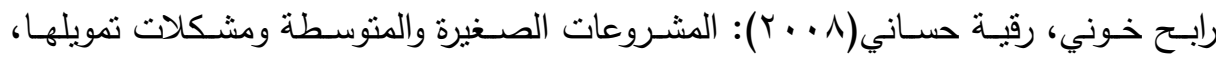

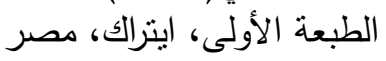

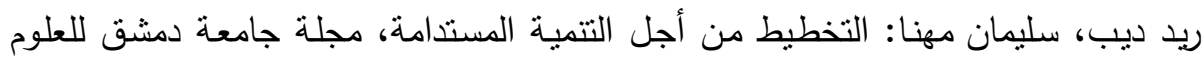
الهندسية، الجمهورية العربية السورية، المجلد الخامس والعشرون، العدد الأول

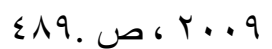

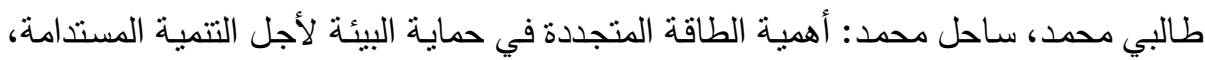

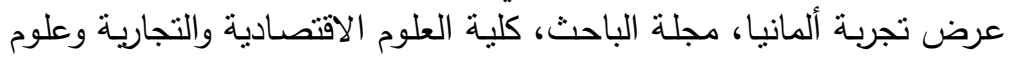

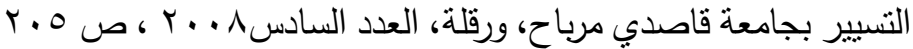

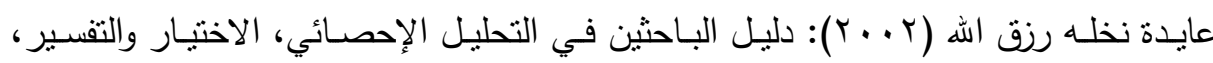
البيان للطباعة الن البان

فتحي السبد عبده أبو السيد أحمد(ه . . ؟): الصناعات الصغيرة ودورها في التتمية، مؤسسة

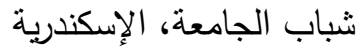

فريدة كافي: بعنوان " الطاقـات المتجددة بين تحديات الواقع ومـأمول المستقبل: التجربـة

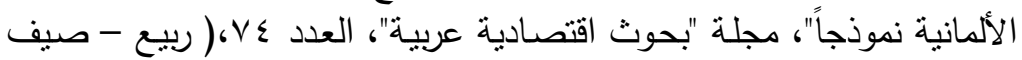

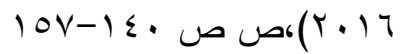

قدي عبد المجيد، منور أوسرير ، محمد حمو ( • • ب): الاقتصساد البيئي، دار الخلدونية للنشر

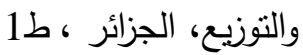

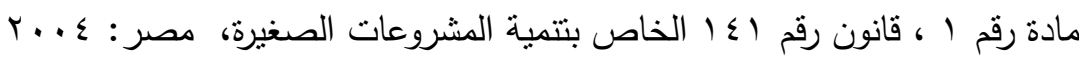

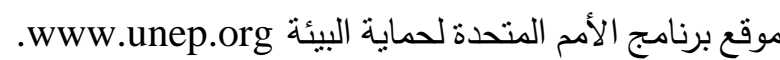

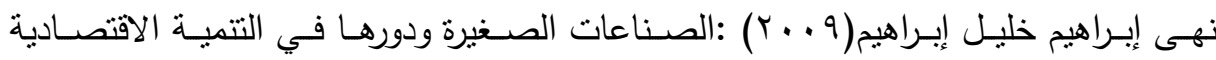
والسياحية، مؤسسة شباب الجامعة، الإسكندرية. 


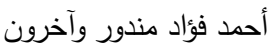

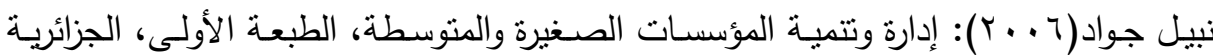

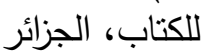

Bebbington, K. J., Gray, R. H., Thompson I, and Walters, D. (1994) "Accountants attitudes and environmentally sensitive accounting", Accounting and Business Research, London; Spring, pp. 51-75.

Agenda 21 : the united nations programmers of action from RIO , June 1992 , chapters $3,4,7$

Andexer Thomas, A Hypothetical Enhanced Renewable Energy Utilization (EREU) Model for Electricity Generation in Thailand, Der Deutschen Bibliothek, Norderstedt Germany, 2008, P 16.

Octave Gélinier et Autres, Développement Durable Pour Une Entreprise Compétitive et Responsable,3eme édition, Esf Editeur, France, 2005, P.22

Paramati, Sudharshan Reddy, Avik Sinha, and Eyup Dogan. "The significance of renewable energy use for economic output and environmental protection: evidence from the Next 11 developing economies." Environmental Science and Pollution Research (2017): 1-15. 
مجلة العلوم البيئية

معهد الدراسات والبحوث البيئة - جامعة عين شمس البئ

\title{
A PROPOSED FRAMEWORK FOR STUDYING OF THE ECONOMIC IMPACT OF THE APPLICATION OF RENEWABLE ENERGY IN SMALL ENTERPRISE FOR ACHIEVING SUSTAINABLE DEVELOPMENT
}

\author{
Mandoor, A. F. ${ }^{(1)}$; Elshahat, Nehal. M. F. ${ }^{(2)}$; Abdelazem, H. A. ${ }^{(3)}$ \\ and Elfalougy, A. M.
}

1-Faculty of Commerce, Ain Shams University 2) The Institute of Environmental Studies \& Research, Ain Shams University 3) Facility of Industrial Education, Suez, University

\begin{abstract}
Small enterprises have the attention of economic and social policy planners in various countries of the developed and developing world alike, because they have an important role in achieving economic and social development. The energy sector plays an important role in achieving development. Energy is a key factor in supporting the production process and achieving economic development, which provides job opportunities and helps improve the standard of living in general.

The study aimed at developing a proposed framework for studying the economic impact of the application of renewable energy in small projects to achieve sustainable development, showing the economic, social, environmental and technological benefits resulting from the application of renewable energy in small projects. An applied case study of a solar-powered plant and a comparison with a diesel generator system has been used. The study reached several results, the most important of which are: (The prominent role of small projects in the economies of developing countries, especially in achieving sustainable development through contributing to the achievement of economic, social and environmental goals.) Reliance on renewable sources of 376

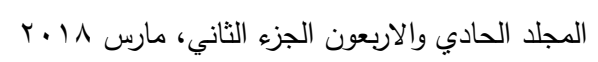


energy is a promising alternative to traditional energy. To conventional energy sources and reduce pollution resulting from conventional energy use). The study recommends the need to pay attention to small projects because they have an effective role in economic, social and environmental, as well as interest in renewable energy sources as a means to support energy security in Egypt, especially with the entry of traditional sources and the increase in the value of support allocations allocated to these sources. 